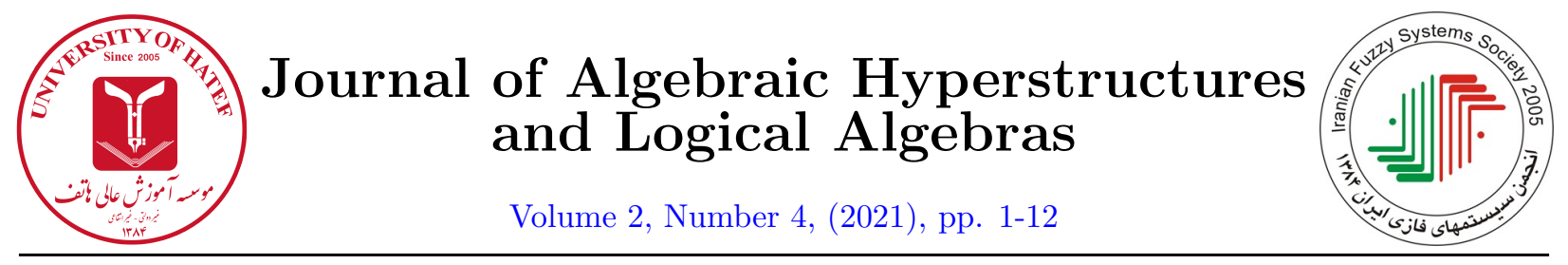

\title{
Implication Zroupoids and Birkhoff systems
}

\author{
J.M. Cornejo ${ }^{1}$ and H.P. Sankappanavar ${ }^{2}$ \\ ${ }^{1}$ Departamento de Matemática, Universidad Nacional del Sur, Alem 1253, Bahía Blanca, Argentina, INMABB - \\ CONICET \\ ${ }^{2}$ Hanamantagouda P. Sankappanavar, Department of Mathematics, State University of New York, New Paltz, New \\ York 12561, U.S.A.
}

jmcornejo@uns.edu.ar,sankapph@newpaltz.edu

\begin{abstract}
An algebra $\mathbf{A}=\langle A, \rightarrow, 0\rangle$, where $\rightarrow$ is binary and 0 is a constant, is called an implication zroupoid ( $\mathcal{I}$-zroupoid, for short) if A satisfies the identities: $(x \rightarrow y) \rightarrow z \approx\left[\left(z^{\prime} \rightarrow x\right) \rightarrow(y \rightarrow\right.$ $\left.z)^{\prime}\right]^{\prime}$, where $x^{\prime}:=x \rightarrow 0$, and $0^{\prime \prime} \approx 0$. These algebras generalize De Morgan algebras and $\vee$-semilattices with zero. Let $\mathcal{I}$ denote the variety of implication zroupoids. The investigations into the structure of $\mathcal{I}$ and of the lattice of subvarieties of $\mathcal{I}$, begun in 2012, have continued in several papers (see the Bibliography at the end of the paper). The present paper is a sequel to that series of papers and is devoted to making further contributions to the theory of implication zroupoids. The main purpose of this paper is to prove that if $\mathbf{A}$ is an algebra in the variety $\mathcal{I}$, then the derived algebra $\mathbf{A}_{m j}:=\langle A ; \wedge, \vee\rangle$, where $a \wedge b:=\left(a \rightarrow b^{\prime}\right)^{\prime}$ and $a \vee b:=\left(a^{\prime} \wedge b^{\prime}\right)^{\prime}$, satisfies the Birkhoff's identity (BR): $x \wedge(x \vee y) \approx x \vee(x \wedge y)$. As a consequence, the implication zroupoids $\mathbf{A}$ whose derived algebras $\mathbf{A}_{m j}$ are Birkhoff systems are characterized. Another interesting consequence of the main result is that there are bisemigroups that are not bisemilattices but satisfy the Birkhoff's identity, which leads us naturally to define the variety of "Birkhoff bisemigroups" as bisemigroups satisfying the Birkhoff identity, as a generalization of Birkhoff systems. The paper concludes with some open problems.
\end{abstract}

\section{Article Information}

Corresponding Author:

H.P. Sankappanavar;

Received: May 2020;

Accepted: Invited paper;

Paper type: Original.

\section{Keywords:}

Implication zroupoid, symmetric implication zroupoid, bisemigroup, Birkhoff identity, Birkhoff system, implication semigroup, Birkhoff bisemigroup.

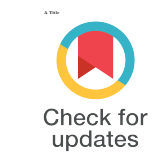

\section{Introduction}

An algebra $\mathbf{A}=\langle A, \rightarrow, 0\rangle$, where $\rightarrow$ is binary and 0 is a constant, is called an implication zroupoid ( $\mathcal{I}$ zroupoid, for short) if $\mathbf{A}$ satisfies the following identities:

(1) $\quad(x \rightarrow y) \rightarrow z \approx\left[\left(z^{\prime} \rightarrow x\right) \rightarrow(y \rightarrow z)^{\prime}\right]^{\prime}$, where $x^{\prime}:=x \rightarrow 0$,

(2) $\quad 0^{\prime \prime} \approx 0$.

https://doi.org/10.52547/HATEF.JAHLA.2.4.1 
Let $\mathcal{I}$ denote the variety of implication zroupoids.

These algebras generalize De Morgan algebras and $\vee$-semilattices with zero. For more details on the motivation leading to these algebras, we refer the reader to [20]] (or the relevant papers mentioned at the end of this paper).

The investigations into the structure of the lattice of subvarieties of $\mathcal{I}$, begun in [20], have continued in

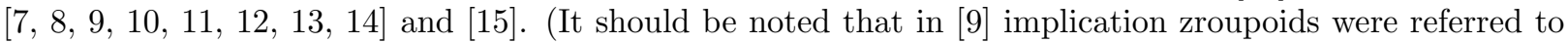
as "implicator groupoids".) The present paper is a sequel to this series of papers and is devoted to making further contributions to the theory of implication zroupoids.

Throughout this paper we use the following definitions:

$$
\text { (M) } \quad x \wedge y:=\left(x \rightarrow y^{\prime}\right)^{\prime} \quad \text { and } \quad(\mathrm{J}) \quad x \vee y:=\left(x^{\prime} \wedge y^{\prime}\right)^{\prime} .
$$

With each $\mathbf{A} \in \mathcal{I}$, we associate the following algebras:

$$
\mathbf{A}^{\mathbf{m j}}:=\langle A, \wedge, \vee, 0\rangle \quad \text { and } \quad \mathbf{A}_{\mathbf{m j}}:=\langle A, \wedge, \vee\rangle .
$$

Theorem 1.1. [III, Corollary 4.6] If $\mathbf{A} \in \mathcal{I}$, then $\langle A, \wedge\rangle$ and $\langle A, \vee\rangle$ are semigroups. Hence, $\mathbf{A}_{\mathbf{m j}}$ is a bisemigroup.

Two of the important subvarieties of $\mathcal{I}$ are: $\mathcal{I}_{2,0}$ and $\mathcal{M C}$ which are defined relative to $\mathcal{I}$, respectively, by the following identities:

$$
\begin{gathered}
x^{\prime \prime} \approx x . \\
x \wedge y \approx y \wedge x .
\end{gathered}
$$

Definition 1.2. Members of the variety $\mathcal{I}_{2,0}$ are called involutive $\mathcal{I}$-zroupoids, and members of $\mathcal{M C}$ are called meet-commutative $\mathcal{I}$-zroupoids. An algebra $\mathbf{A} \in \mathcal{I}$ is symmetric if $\mathbf{A}$ is both involutive and meetcommutative.

Let $\mathcal{S}$ denote the variety of symmetric $\mathcal{I}$-zroupoids. Thus, $\mathcal{S}=\mathcal{I}_{2,0} \cap \mathcal{M C}$. The identity

$$
\text { (BR) } x \wedge(x \vee y) \approx x \vee(x \wedge y) .
$$

is called the Birkhoff's identity. This identity, a weakened form of the absorption identities, was introduced by Birkhoff in 1948. In fact, Birkhoff asked in [3, Problem 7] for an investigation of algebras satisfying the lattice identities without absorption identities but with the identity (BR), which led to the following notion:

Definition 1.3. A Birkhoff system is a bisemilattice satisfying the Birkhoff's identity (BR).

Indeed, in response to Birkhoff's problem, there have been a series of papers in the literature revealing the structure of the lattice of subvarieties of the variety of Birkhoff systems; for example, see [i6] and the references therein. More recently, it was proved in [ 9 , Theorem 7.3] that if $\mathbf{A} \in \mathcal{S}$, then $\mathbf{A}^{m j}$ is a distributive Birkhoff system, from which it immediately follows that $\mathbf{A}_{m j}$ is a distributive Birkhoff system-a result which will be strengthened in this paper.

The main purpose of this paper is to prove the following theorem:

Theorem 1.4. If $\mathbf{A} \in \mathcal{I}$, then the derived algebra $\mathbf{A}_{m j}$ satisfies the Birkhoff's identity:

$$
\text { (BR) } \mathrm{x} \wedge(\mathrm{x} \vee \mathrm{y}) \approx \mathrm{x} \vee(\mathrm{x} \wedge \mathrm{y}) .
$$

As a consequence, the implication zroupoids $\mathbf{A}$ whose derived algebras $\mathbf{A}_{m j}$ are Birkhoff systems are characterized. Another interesting consequence of Theorem $\mathbb{L} \mathbb{4}$ is that there are bisemigroups that are not bisemilattices but satisfy the Birkhoff's identity. This leads us naturally to define the variety of "Birkhoff bisemigroups" as bisemigroups satisfying the Birkhoff identity, as a generalization of Birkhoff systems. The paper concludes with some open problems. 


\section{Preliminaries}

In this section we present some preliminary results that will be useful later.

Lemma 2.1. [20, Theorem 8.15] The following identities are equivalent in the variety $\mathcal{I}$ :

(a) $0^{\prime} \rightarrow x \approx x$,

(b) $x^{\prime \prime} \approx x$,

(c) $\left(x \rightarrow x^{\prime}\right)^{\prime} \approx x$,

(d) $x^{\prime} \rightarrow x \approx x$.

The following theorem is proved in [9, Theorem 7.3].

Theorem 2.2. Let $\mathbf{A} \in \mathcal{S}$. Then $\mathbf{A}_{m j}$ satisfies:

(a) $x \wedge x \approx x$,

(b) $x \vee x \approx x$,

(c) $x \vee y \approx y \vee x$,

(d) $x \wedge(x \vee y) \approx x \vee(x \wedge y)$.

Lemma 2.3. Let $\mathbf{A} \in \mathcal{I}_{2,0}$. Then $\mathbf{A}$ satisfies:

1. $x^{\prime} \rightarrow 0^{\prime} \approx 0 \rightarrow x$,

2. $0 \rightarrow x^{\prime} \approx x \rightarrow 0^{\prime}$,

3. $\left(x \rightarrow 0^{\prime}\right) \rightarrow(y \rightarrow z) \approx((0 \rightarrow x) \rightarrow y) \rightarrow z$,

4. $(0 \rightarrow x) \rightarrow(0 \rightarrow y) \approx x \rightarrow(0 \rightarrow y)$,

5. $0 \rightarrow(x \rightarrow y) \approx x \rightarrow(0 \rightarrow y)$,

6. $0 \rightarrow\left(x^{\prime} \rightarrow y\right)^{\prime} \approx x \rightarrow\left(0 \rightarrow y^{\prime}\right)$,

7. $(x \rightarrow y) \rightarrow(y \rightarrow z) \approx\left(0 \rightarrow x^{\prime}\right) \rightarrow(y \rightarrow z)$,

8. $((x \rightarrow y) \rightarrow z) \rightarrow(z \rightarrow u) \approx(0 \rightarrow x) \rightarrow((y \rightarrow z) \rightarrow(z \rightarrow u))$,

9. $x \rightarrow y \approx x \rightarrow(x \rightarrow y)$,

10. $(y \rightarrow x) \rightarrow y \approx(0 \rightarrow x) \rightarrow y$,

11. $(x \rightarrow y)^{\prime} \rightarrow(0 \rightarrow x)^{\prime} \approx y^{\prime} \rightarrow x^{\prime}$,

12. $(x \rightarrow y)^{\prime} \rightarrow y \approx x \rightarrow y$,

13. $\left[x \rightarrow(y \rightarrow x)^{\prime}\right]^{\prime} \approx(x \rightarrow y) \rightarrow x$,

14. $x \rightarrow((0 \rightarrow x) \rightarrow y) \approx x \rightarrow y$,

15. $x \rightarrow\left(y \rightarrow x^{\prime}\right) \approx y \rightarrow x^{\prime}$,

16. $(x \rightarrow y) \rightarrow y^{\prime} \approx y \rightarrow(x \rightarrow y)^{\prime}$,

17. $((x \rightarrow y) \rightarrow(z \rightarrow x)) \rightarrow u \approx\left(y \rightarrow 0^{\prime}\right) \rightarrow((z \rightarrow x) \rightarrow u)$,

18. $(z \rightarrow x) \rightarrow(y \rightarrow z) \approx(0 \rightarrow x) \rightarrow(y \rightarrow z)$,

19. $\left(x \rightarrow y^{\prime}\right)^{\prime} \rightarrow z \approx x \rightarrow(y \rightarrow z)$, 
20. $0 \rightarrow\left(x \rightarrow y^{\prime}\right)^{\prime} \approx 0 \rightarrow\left(x^{\prime} \rightarrow y\right)$

21. $0 \rightarrow(0 \rightarrow x)^{\prime} \approx 0 \rightarrow x^{\prime}$,

22. $\left[x^{\prime} \rightarrow(0 \rightarrow y)\right]^{\prime} \approx(0 \rightarrow x) \rightarrow(0 \rightarrow y)^{\prime}$.

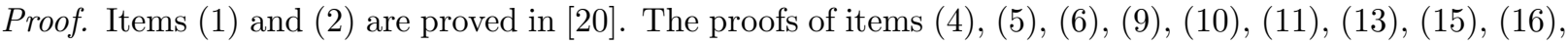

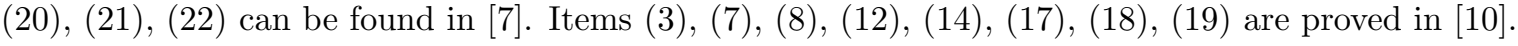

\section{$3 \quad \mathcal{I}_{2,0}$ and the Birkhoff Identity}

In this section we prove a special case of our main result that if $\mathbf{A} \in \mathcal{I}_{2,0}$, then the bisemigroup $\mathbf{A}_{m j}$ satisfies (BR). This result will play a crucial role in the proof of the main Theorem in the next section.

Lemma 3.1. Let $\mathbf{A} \in \mathcal{I}_{2,0}$. Then

1. $((x \rightarrow(0 \rightarrow y)) \rightarrow z) \rightarrow u \approx(0 \rightarrow x) \rightarrow\left(\left(0 \rightarrow y^{\prime}\right) \rightarrow(z \rightarrow u)\right)$,

2. $((0 \rightarrow x) \rightarrow y) \rightarrow z \approx(x \rightarrow y) \rightarrow(y \rightarrow z)$,

3. $((x \rightarrow y) \rightarrow z) \rightarrow(z \rightarrow u) \approx(0 \rightarrow x) \rightarrow\left(\left(0 \rightarrow y^{\prime}\right) \rightarrow(z \rightarrow u)\right)$,

4. $((x \rightarrow y) \rightarrow z) \rightarrow x^{\prime} \approx(y \rightarrow z) \rightarrow x^{\prime}$,

5. $\left[x \rightarrow\left[\left(0 \rightarrow y^{\prime}\right) \rightarrow(z \rightarrow u)\right]\right] \rightarrow\left[(0 \rightarrow y) \rightarrow\left[(z \rightarrow u) \rightarrow(0 \rightarrow(x \rightarrow y))^{\prime}\right]\right] \approx(z \rightarrow u) \rightarrow(0 \rightarrow(x \rightarrow y))^{\prime}$,

6. $(x \rightarrow(0 \rightarrow y)) \rightarrow z \approx(z \rightarrow(x \rightarrow y)) \rightarrow z$,

7. $[0 \rightarrow(x \rightarrow(y \rightarrow z))] \rightarrow u \approx\left(0 \rightarrow x^{\prime}\right) \rightarrow((0 \rightarrow(y \rightarrow z)) \rightarrow u)$,

8. $(x \rightarrow y) \rightarrow((0 \rightarrow y) \rightarrow z) \approx(x \rightarrow y) \rightarrow z$,

9. $(x \rightarrow y) \rightarrow((z \rightarrow y) \rightarrow(u \rightarrow z)) \approx(x \rightarrow y) \rightarrow(u \rightarrow z)$,

10. $\left[(0 \rightarrow y) \rightarrow z^{\prime}\right] \rightarrow u \approx\left(y \rightarrow z^{\prime}\right) \rightarrow\left(z^{\prime} \rightarrow u\right)$,

11. $(0 \rightarrow x) \rightarrow((y \rightarrow x) \rightarrow z) \approx(y \rightarrow x) \rightarrow z$,

12. $x^{\prime} \rightarrow(0 \rightarrow(y \rightarrow z))^{\prime} \approx x^{\prime} \rightarrow(x \rightarrow(y \rightarrow z))^{\prime}$,

13. $0 \rightarrow(x \rightarrow(y \rightarrow z)) \approx 0 \rightarrow\left(\left(x^{\prime} \rightarrow y\right) \rightarrow z\right)$,

14. $0 \rightarrow[x \rightarrow((y \rightarrow z) \rightarrow u)] \approx 0 \rightarrow[((x \rightarrow y) \rightarrow z) \rightarrow u]$,

15. $\left(x \rightarrow y^{\prime}\right) \rightarrow\left[y^{\prime} \rightarrow\left(0 \rightarrow x^{\prime}\right)^{\prime}\right] \approx y^{\prime} \rightarrow\left(0 \rightarrow x^{\prime}\right)^{\prime}$,

16. $\left[x \rightarrow\left(x^{\prime} \rightarrow y\right)^{\prime}\right]^{\prime} \approx x^{\prime} \rightarrow\left(0 \rightarrow y^{\prime}\right)^{\prime}$.

Proof. Let $a, b, c, d \in A$.

(四)

$$
\begin{aligned}
& (0 \rightarrow a) \rightarrow\left(\left(0 \rightarrow b^{\prime}\right) \rightarrow(c \rightarrow d)\right)
\end{aligned}
$$

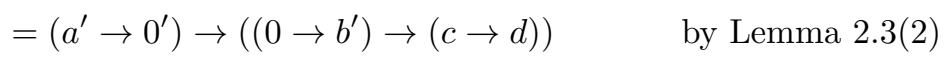

$$
\begin{aligned}
& =\left[\left(0 \rightarrow a^{\prime}\right) \rightarrow\left(0 \rightarrow b^{\prime}\right)\right] \rightarrow(c \rightarrow d) \quad \text { by Lemma [2.3(13) } \\
& =\left[0 \rightarrow\left(a^{\prime} \rightarrow b^{\prime}\right)\right] \rightarrow(c \rightarrow d) \quad \text { by Lemma [2.3(四) and (回) } \\
& =\left[0 \rightarrow(a \rightarrow b)^{\prime}\right] \rightarrow(c \rightarrow d) \\
& =\left[(a \rightarrow b) \rightarrow 0^{\prime}\right] \rightarrow(c \rightarrow d) \\
& =[[0 \rightarrow(a \rightarrow b)] \rightarrow c] \rightarrow d \\
& =[[a \rightarrow(0 \rightarrow b)] \rightarrow c] \rightarrow d \\
& \text { by Lemma [.3. (19) and (6) }
\end{aligned}
$$

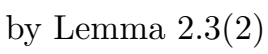

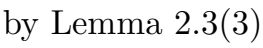

$$
\begin{aligned}
& \text { by Lemma [2.3(回) }
\end{aligned}
$$


(匹)

$$
\begin{aligned}
& ((0 \rightarrow a) \rightarrow b) \rightarrow c \\
= & \left(a \rightarrow 0^{\prime}\right) \rightarrow(b \rightarrow c) \\
= & \left(0 \rightarrow a^{\prime}\right) \rightarrow(b \rightarrow c) \\
= & (a \rightarrow b) \rightarrow(b \rightarrow c)
\end{aligned}
$$

by Lemma $[23(\mathbb{Z})$

by Lemma $[2.3(\mathbb{\square})$.

(B)

$$
\begin{aligned}
& ((a \rightarrow b) \rightarrow c) \rightarrow(c \rightarrow d)
\end{aligned}
$$

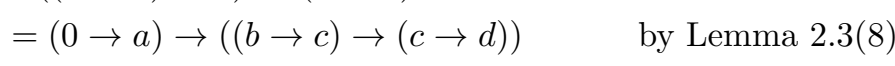

$$
\begin{aligned}
& =(0 \rightarrow a) \rightarrow\left(\left(0 \rightarrow b^{\prime}\right) \rightarrow(c \rightarrow d)\right) \quad \text { by Lemma [2.3]([) }
\end{aligned}
$$

(四)

$$
\begin{aligned}
& ((a \rightarrow b) \rightarrow c) \rightarrow a^{\prime} \\
& =\left[\left(a^{\prime \prime} \rightarrow(a \rightarrow b)\right) \rightarrow\left(c \rightarrow a^{\prime}\right)^{\prime}\right]^{\prime} \quad \text { by (I) } \\
& =\left[(a \rightarrow(a \rightarrow b)) \rightarrow\left(c \rightarrow a^{\prime}\right)^{\prime}\right]^{\prime} \\
& =\left[(a \rightarrow b) \rightarrow\left(c \rightarrow a^{\prime}\right)^{\prime}\right]^{\prime} \\
& =\left[\left(a^{\prime \prime} \rightarrow b\right) \rightarrow\left(c \rightarrow a^{\prime}\right)^{\prime}\right]^{\prime} \\
& =(b \rightarrow c) \rightarrow a^{\prime}
\end{aligned}
$$

(回)

$$
\begin{aligned}
& (c \rightarrow d) \rightarrow(0 \rightarrow(a \rightarrow b))^{\prime} \\
= & {[((0 \rightarrow(a \rightarrow b)) \rightarrow c] \rightarrow d] \rightarrow(0 \rightarrow(a \rightarrow b))^{\prime} }
\end{aligned}
$$

by (ब) with $x:=0 \rightarrow(a \rightarrow b), y:=c, z:=d$

$=[[(a \rightarrow(0 \rightarrow b)) \rightarrow c] \rightarrow d] \rightarrow(0 \rightarrow(a \rightarrow b))^{\prime} \quad$ by Lemma [2.3 (回)

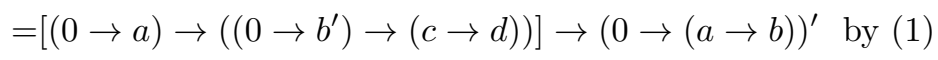

$=\left[a \rightarrow\left(\left(0 \rightarrow b^{\prime}\right) \rightarrow(c \rightarrow d)\right)\right] \rightarrow\left[\left(\left(0 \rightarrow b^{\prime}\right) \rightarrow(c \rightarrow d)\right) \rightarrow(0 \rightarrow(a \rightarrow b))^{\prime}\right]$

by (四) with $x:=a, y:=\left(0 \rightarrow b^{\prime}\right) \rightarrow(c \rightarrow d), z:=(0 \rightarrow(a \rightarrow b))^{\prime}$

$=\left[a \rightarrow\left(\left(0 \rightarrow b^{\prime}\right) \rightarrow(c \rightarrow d)\right)\right] \rightarrow\left[\left(b^{\prime} \rightarrow(c \rightarrow d)\right) \rightarrow\left((c \rightarrow d) \rightarrow(0 \rightarrow(a \rightarrow b))^{\prime}\right)\right]$

by (四) with $x:=b^{\prime}, y:=c \rightarrow d, z:=(0 \rightarrow(a \rightarrow b))^{\prime}$

$=\left[a \rightarrow\left(\left(0 \rightarrow b^{\prime}\right) \rightarrow(c \rightarrow d)\right)\right] \rightarrow\left\{(0 \rightarrow b) \rightarrow\left[\left(0 \rightarrow 0^{\prime}\right) \rightarrow\left[(c \rightarrow d) \rightarrow(0 \rightarrow(a \rightarrow b))^{\prime}\right]\right]\right\}$

by (B) with $x:=b, y:=0, z:=c \rightarrow d, u:=(0 \rightarrow(a \rightarrow b))^{\prime}$

$=\left[a \rightarrow\left(\left(0 \rightarrow b^{\prime}\right) \rightarrow(c \rightarrow d)\right)\right] \rightarrow\left\{(0 \rightarrow b) \rightarrow\left[\left(0^{\prime \prime} \rightarrow 0^{\prime}\right) \rightarrow\left[(c \rightarrow d) \rightarrow(0 \rightarrow(a \rightarrow b))^{\prime}\right]\right]\right\}$

$=\left[a \rightarrow\left(\left(0 \rightarrow b^{\prime}\right) \rightarrow(c \rightarrow d)\right)\right] \rightarrow\left\{(0 \rightarrow b) \rightarrow\left[0^{\prime} \rightarrow\left[(c \rightarrow d) \rightarrow(0 \rightarrow(a \rightarrow b))^{\prime}\right]\right]\right\}$ by Lemma $[].(\mathrm{d})$

$=\left[a \rightarrow\left(\left(0 \rightarrow b^{\prime}\right) \rightarrow(c \rightarrow d)\right)\right] \rightarrow\left\{(0 \rightarrow b) \rightarrow\left[(c \rightarrow d) \rightarrow(0 \rightarrow(a \rightarrow b))^{\prime}\right]\right\}$ by Lemma $[2].($ a $)$

(6)

$$
\begin{aligned}
& (a \rightarrow(0 \rightarrow b)) \rightarrow c \\
& =(0 \rightarrow(a \rightarrow b)) \rightarrow c \quad \text { by Lemma [2.3(回) } \\
& =(c \rightarrow(a \rightarrow b)) \rightarrow c \quad \text { by Lemma [..3(四) }
\end{aligned}
$$


(घ)

$$
\begin{aligned}
& \left(0 \rightarrow a^{\prime}\right) \rightarrow((0 \rightarrow(b \rightarrow c)) \rightarrow d) \\
= & \left(a \rightarrow 0^{\prime}\right) \rightarrow((0 \rightarrow(b \rightarrow c)) \rightarrow d) \\
= & {[(0 \rightarrow a) \rightarrow(0 \rightarrow(b \rightarrow c))] \rightarrow d } \\
= & {[0 \rightarrow(a \rightarrow(b \rightarrow c))] \rightarrow d }
\end{aligned}
$$

(区)

$$
\begin{aligned}
& (a \rightarrow b) \rightarrow((0 \rightarrow b) \rightarrow c) \\
& =\left[\left[((0 \rightarrow b) \rightarrow c)^{\prime} \rightarrow a\right] \rightarrow[b \rightarrow((0 \rightarrow b) \rightarrow c)]^{\prime}\right]^{\prime} \quad \text { by }(\mathrm{I}) \\
& =\left[\left[((0 \rightarrow b) \rightarrow c)^{\prime} \rightarrow a\right] \rightarrow[b \rightarrow c]^{\prime}\right]^{\prime} \quad \text { by Lemma [.3. (प4 } \\
& =\left[\left[((c \rightarrow b) \rightarrow c)^{\prime} \rightarrow a\right] \rightarrow[b \rightarrow c]^{\prime}\right]^{\prime} \quad \text { by Lemma [2.3(四) } \\
& =\left[\left[\left(c \rightarrow(b \rightarrow c)^{\prime}\right)^{\prime \prime} \rightarrow a\right] \rightarrow[b \rightarrow c]^{\prime}\right]^{\prime} \quad \text { by Lemma [2.3 ([ए3) } \\
& =\left[\left[\left(c \rightarrow(b \rightarrow c)^{\prime}\right) \rightarrow a\right] \rightarrow[b \rightarrow c]^{\prime}\right]^{\prime} \quad \text { by }\left(\mathrm{I}_{2,0}\right) \\
& =\left[[b \rightarrow c] \rightarrow\left(c \rightarrow(b \rightarrow c)^{\prime}\right)\right] \rightarrow\left[a \rightarrow[b \rightarrow c]^{\prime}\right]^{\prime} \quad \text { by (I) and }\left(\mathrm{I}_{2,0}\right) \\
& =\left[c \rightarrow(b \rightarrow c)^{\prime}\right] \rightarrow\left[a \rightarrow[b \rightarrow c]^{\prime}\right]^{\prime} \quad \text { by Lemma [.3. (1.5) } \\
& =\left[(b \rightarrow c) \rightarrow c^{\prime}\right] \rightarrow\left[a \rightarrow[b \rightarrow c]^{\prime}\right]^{\prime} \\
& =\left[\left(c^{\prime} \rightarrow a\right) \rightarrow[b \rightarrow c]^{\prime}\right]^{\prime} \\
& =(a \rightarrow b) \rightarrow c \\
& \text { by Lemma } 2.3 \text { (106) } \\
& \text { by }\left(\mathrm{I}_{2,0}\right) \text { and }(\mathrm{I}) \\
& \text { by (I) }
\end{aligned}
$$

(四)

$$
\begin{aligned}
& (a \rightarrow b) \rightarrow((c \rightarrow b) \rightarrow(d \rightarrow c)) \\
& =(a \rightarrow b) \rightarrow\left[\left[(d \rightarrow c)^{\prime} \rightarrow c\right] \rightarrow[b \rightarrow(d \rightarrow c)]^{\prime}\right]^{\prime} \quad \text { by (I) } \\
& =(a \rightarrow b) \rightarrow\left[(d \rightarrow c) \rightarrow[b \rightarrow(d \rightarrow c)]^{\prime}\right]^{\prime} \quad \text { by Lemma ए2.3(एव) } \\
& =(a \rightarrow b) \rightarrow[[(d \rightarrow c) \rightarrow b] \rightarrow(d \rightarrow c)] \quad \text { by Lemma [2.3]([.3]) } \\
& =(a \rightarrow b) \rightarrow[[0 \rightarrow b] \rightarrow(d \rightarrow c)] \\
& =(a \rightarrow b) \rightarrow(d \rightarrow c)
\end{aligned}
$$

(एण)

$$
\begin{aligned}
& {[(0 \rightarrow b) \rightarrow(c \rightarrow 0)] \rightarrow d} \\
& =\left(b \rightarrow 0^{\prime}\right) \rightarrow((c \rightarrow 0) \rightarrow d) \quad \text { by Lemma [2](마) } \\
& =\left(0 \rightarrow b^{\prime}\right) \rightarrow((c \rightarrow 0) \rightarrow d) \quad \text { by Lemma [2.](西) } \\
& =\left(b \rightarrow c^{\prime}\right) \rightarrow\left(c^{\prime} \rightarrow d\right) \quad \text { by Lemma } 2.3(7) \text {. }
\end{aligned}
$$

(ㅁ)

$$
\begin{aligned}
& (0 \rightarrow a) \rightarrow((b \rightarrow a) \rightarrow c) \\
& =\left(a^{\prime} \rightarrow 0^{\prime}\right) \rightarrow((b \rightarrow a) \rightarrow c) \quad \text { by Lemma [..3( (घ) and }\left(\mathrm{I}_{2,0}\right) \\
& =\left[\left(0 \rightarrow a^{\prime}\right) \rightarrow(b \rightarrow a)\right] \rightarrow c \quad \text { by Lemma [2.3(B) } \\
& =\left[\left(a \rightarrow a^{\prime}\right) \rightarrow(b \rightarrow a)\right] \rightarrow c \quad \text { by Lemma [.](피) } \\
& \left.=\left[a^{\prime} \rightarrow(b \rightarrow a)\right] \rightarrow c \quad \text { by Lemma } 2\right](\mathrm{d}) \text { and }\left(\mathrm{I}_{2,0}\right)
\end{aligned}
$$

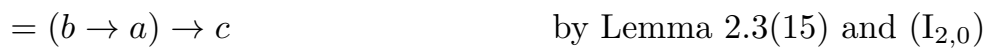


(ए2)

$$
\begin{aligned}
& a^{\prime} \rightarrow(a \rightarrow(b \rightarrow c))^{\prime} \\
= & {[(a \rightarrow(b \rightarrow c)) \rightarrow a]^{\prime} \rightarrow[0 \rightarrow(a \rightarrow(b \rightarrow c))]^{\prime} }
\end{aligned}
$$

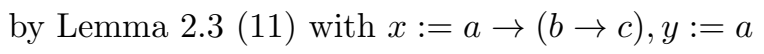

$=[(b \rightarrow(0 \rightarrow c)) \rightarrow a]^{\prime} \rightarrow[0 \rightarrow(a \rightarrow(b \rightarrow c))]^{\prime} \quad$ by $($ [G $)$

$=\left[(0 \rightarrow b) \rightarrow\left(\left(0 \rightarrow c^{\prime}\right) \rightarrow a^{\prime}\right)\right] \rightarrow[0 \rightarrow(a \rightarrow(b \rightarrow c))]^{\prime}$

by (四) with $x:=b, y:=c, z:=a, u:=0$

$=\left[(0 \rightarrow b) \rightarrow\left(\left(0 \rightarrow c^{\prime}\right) \rightarrow a^{\prime}\right)\right] \rightarrow\left[\left(0 \rightarrow a^{\prime}\right) \rightarrow(0 \rightarrow(b \rightarrow c))^{\prime}\right]$ by $(\mathbb{G})$ with $u:=0$

$=\left(b \rightarrow\left(\left(0 \rightarrow c^{\prime}\right) \rightarrow a^{\prime}\right)\right) \rightarrow\left[\left(\left(0 \rightarrow c^{\prime}\right) \rightarrow a^{\prime}\right) \rightarrow\left[\left(0 \rightarrow a^{\prime}\right) \rightarrow(0 \rightarrow(b \rightarrow c))^{\prime}\right]\right]$

by (四) with $x:=b, y:=\left(0 \rightarrow c^{\prime}\right) \rightarrow a^{\prime}, z:=\left(0 \rightarrow a^{\prime}\right) \rightarrow(0 \rightarrow(b \rightarrow c))^{\prime}$

$=\left(b \rightarrow\left(\left(0 \rightarrow c^{\prime}\right) \rightarrow a^{\prime}\right)\right) \rightarrow\left[\left(\left(0 \rightarrow c^{\prime}\right) \rightarrow a^{\prime}\right) \rightarrow(0 \rightarrow(b \rightarrow c))^{\prime}\right]$

by (四) with $x:=0 \rightarrow c^{\prime}, y:=a^{\prime}, z:=0, u:=0 \rightarrow(b \rightarrow c)$

$=\left(b \rightarrow\left(\left(0 \rightarrow c^{\prime}\right) \rightarrow a^{\prime}\right)\right) \rightarrow\left[\left(c^{\prime} \rightarrow(a \rightarrow 0)\right) \rightarrow\left[(a \rightarrow 0) \rightarrow(0 \rightarrow(b \rightarrow c))^{\prime}\right]\right]$

by (四) with $y:=c^{\prime}, z:=a, u:=(0 \rightarrow(b \rightarrow c))^{\prime}$

$=\left(b \rightarrow\left(\left(0 \rightarrow c^{\prime}\right) \rightarrow a^{\prime}\right)\right) \rightarrow\left[\left((c \rightarrow 0) \rightarrow a^{\prime}\right) \rightarrow\left[a^{\prime} \rightarrow(0 \rightarrow(b \rightarrow c))^{\prime}\right]\right]$

$=\left(b \rightarrow\left(\left(0 \rightarrow c^{\prime}\right) \rightarrow a^{\prime}\right)\right) \rightarrow\left[(0 \rightarrow c) \rightarrow\left[\left(0 \rightarrow 0^{\prime}\right) \rightarrow\left(a^{\prime} \rightarrow(0 \rightarrow(b \rightarrow c))^{\prime}\right)\right]\right]$

by (B) with $x:=c, y:=0, z:=a^{\prime}, u:=(0 \rightarrow(b \rightarrow c))^{\prime}$

$=\left(b \rightarrow\left(\left(0 \rightarrow c^{\prime}\right) \rightarrow a^{\prime}\right)\right) \rightarrow\left[(0 \rightarrow c) \rightarrow\left[\left(0^{\prime \prime} \rightarrow 0^{\prime}\right) \rightarrow\left(a^{\prime} \rightarrow(0 \rightarrow(b \rightarrow c))^{\prime}\right)\right]\right]$

$=\left(b \rightarrow\left(\left(0 \rightarrow c^{\prime}\right) \rightarrow a^{\prime}\right)\right) \rightarrow\left[(0 \rightarrow c) \rightarrow\left[0^{\prime} \rightarrow\left(a^{\prime} \rightarrow(0 \rightarrow(b \rightarrow c))^{\prime}\right)\right]\right]$

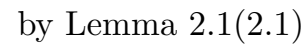

$=\left(b \rightarrow\left(\left(0 \rightarrow c^{\prime}\right) \rightarrow a^{\prime}\right)\right) \rightarrow\left[(0 \rightarrow c) \rightarrow\left(a^{\prime} \rightarrow(0 \rightarrow(b \rightarrow c))^{\prime}\right)\right]$

by Lemma [.] (ए.])

$=(a \rightarrow 0) \rightarrow(0 \rightarrow(b \rightarrow c))^{\prime} \quad$ by (回) with $x:=b, y:=c, z:=a, u:=0$

$=a^{\prime} \rightarrow(0 \rightarrow(b \rightarrow c))^{\prime}$.

(피)

$$
\begin{aligned}
& 0 \rightarrow(a \rightarrow(b \rightarrow c)) \\
& =0 \rightarrow\left[\left(a \rightarrow b^{\prime}\right)^{\prime} \rightarrow c\right] \quad \text { by Lemma } 2.3(\mathbb{L Q} \text {. }
\end{aligned}
$$

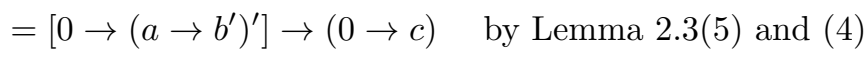

$$
\begin{aligned}
& =\left[0 \rightarrow\left(a^{\prime} \rightarrow b\right)\right] \rightarrow(0 \rightarrow c) \quad \text { [23](201) } \\
& =0 \rightarrow\left(\left(a^{\prime} \rightarrow b\right) \rightarrow c\right) \quad \text { ए.3 (四) and (回) }
\end{aligned}
$$

(ㅁ4)

$$
\begin{aligned}
& 0 \rightarrow[a \rightarrow((b \rightarrow c) \rightarrow d)] \\
& =0 \rightarrow\left[\left(a^{\prime} \rightarrow(b \rightarrow c)\right) \rightarrow d\right] \quad \text { by }(\text { [ए3. }) \\
& =\left[0 \rightarrow\left(a^{\prime} \rightarrow(b \rightarrow c)\right)\right] \rightarrow(0 \rightarrow d) \quad \text { by Lemma [2.3(回) and (마) } \\
& =\left[0 \rightarrow\left(\left(a^{\prime \prime} \rightarrow b\right) \rightarrow c\right)\right] \rightarrow(0 \rightarrow d) \quad \text { by }(\mathbb{[ \amalg 3}) \\
& =[0 \rightarrow((a \rightarrow b) \rightarrow c)] \rightarrow(0 \rightarrow d) \\
& =0 \rightarrow[((a \rightarrow b) \rightarrow c) \rightarrow d]
\end{aligned}
$$


(ㅁ.5)

$$
\begin{aligned}
& \left(a \rightarrow b^{\prime}\right) \rightarrow\left[b^{\prime} \rightarrow\left(0 \rightarrow a^{\prime}\right)^{\prime}\right] \\
& =\left(0 \rightarrow a^{\prime}\right) \rightarrow\left[b^{\prime} \rightarrow\left(0 \rightarrow a^{\prime}\right)^{\prime}\right] \quad \text { by Lemma [2.3(प) } \\
& =b^{\prime} \rightarrow\left(0 \rightarrow a^{\prime}\right)^{\prime} \quad \text { by Lemma [2.3(10.5) }
\end{aligned}
$$

(ㅁ)

$$
\begin{aligned}
& {\left[a \rightarrow\left(a^{\prime} \rightarrow b\right)^{\prime}\right]^{\prime}} \\
& =\left[\left(a^{\prime} \rightarrow 0\right) \rightarrow\left(a^{\prime} \rightarrow b\right)^{\prime}\right]^{\prime} \\
& =\left[\left[\left(a^{\prime} \rightarrow b\right)^{\prime \prime} \rightarrow a^{\prime}\right] \rightarrow\left(0 \rightarrow\left(a^{\prime} \rightarrow b\right)^{\prime}\right)^{\prime}\right]^{\prime \prime} \quad \text { by }(\mathrm{I}) \\
& =\left[\left(a^{\prime} \rightarrow b\right) \rightarrow a^{\prime}\right] \rightarrow\left(0 \rightarrow\left(a^{\prime} \rightarrow b\right)^{\prime}\right)^{\prime} \\
& =\left[(0 \rightarrow b) \rightarrow a^{\prime}\right] \rightarrow\left(0 \rightarrow\left(a^{\prime} \rightarrow b\right)^{\prime}\right)^{\prime} \quad \text { by Lemma [.3. (سل]) } \\
& =\left[(0 \rightarrow b) \rightarrow a^{\prime}\right] \rightarrow\left[0 \rightarrow\left(a \rightarrow(0 \rightarrow b)^{\prime}\right)\right]^{\prime} \text { by (四) with } x:=a, y:=0, z:=b, u:=0 \\
& =\left[(0 \rightarrow b) \rightarrow a^{\prime}\right] \rightarrow\left[a \rightarrow\left(0 \rightarrow(0 \rightarrow b)^{\prime}\right)\right]^{\prime} \quad \text { by Lemma [2.3(10) } \\
& =\left[(0 \rightarrow b) \rightarrow a^{\prime}\right] \rightarrow\left[a \rightarrow\left(0 \rightarrow b^{\prime}\right)\right]^{\prime} \quad \text { by Lemma }[2.3(\text { (2⿴囗⿱一一儿口) } \\
& =\left[(0 \rightarrow b) \rightarrow a^{\prime}\right] \rightarrow\left[a^{\prime \prime} \rightarrow\left(0 \rightarrow b^{\prime}\right)\right]^{\prime} \\
& =\left[(0 \rightarrow b) \rightarrow a^{\prime}\right] \rightarrow\left[\left(0 \rightarrow a^{\prime}\right) \rightarrow\left(0 \rightarrow b^{\prime}\right)^{\prime}\right] \text { by Lemma [2.3(एव) } \\
& =\left[(0 \rightarrow b) \rightarrow a^{\prime}\right] \rightarrow\left(0 \rightarrow b^{\prime}\right)^{\prime} \quad \text { by (四) with } x:=0 \rightarrow b, y:=a^{\prime}, z:=0, u:=0 \rightarrow b^{\prime} \\
& =\left(b \rightarrow a^{\prime}\right) \rightarrow\left[a^{\prime} \rightarrow\left(0 \rightarrow b^{\prime}\right)^{\prime}\right] \text { by (四) with } y:=b, z:=a, u:=\left(0 \rightarrow b^{\prime}\right)^{\prime} \\
& =a^{\prime} \rightarrow\left(0 \rightarrow b^{\prime}\right)^{\prime} \quad \text { by (피) with } x:=b, y:=a \text {. }
\end{aligned}
$$

The proof of our main result (Theorem 4.2 ), given in the next section, depends on the following theorem.

Theorem 3.2. Let $\mathbf{A} \in \mathcal{I}_{2,0}$. Then $\mathbf{A}_{m j}$ satisfies the Birkhoff identity.

Proof. Let $a, b \in A$. Then

$$
\begin{aligned}
& a \wedge(a \vee b)=\left(a \rightarrow\left(a^{\prime} \rightarrow b\right)^{\prime}\right)^{\prime} \quad \text { by definition of } \vee \text { and } \wedge \\
& =a^{\prime} \rightarrow\left(0 \rightarrow b^{\prime}\right)^{\prime} \quad \text { by Lemma B.] (प6]) }
\end{aligned}
$$

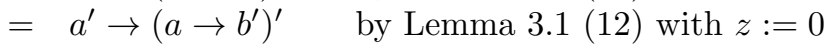

$$
\begin{aligned}
& =\left[a^{\prime} \rightarrow\left(a \rightarrow b^{\prime}\right)^{\prime}\right]^{\prime \prime} \\
& =\left(a^{\prime} \wedge(a \wedge b)^{\prime}\right)^{\prime} \quad \text { by definition of } \wedge \\
& =a \vee(a \wedge b) \quad \text { by definition of } \wedge \text {. }
\end{aligned}
$$

\section{Main theorem}

In this section, the main theorem of this paper is proved. For this we need one more crucial result proved in [110].

Theorem 4.1 (Transfer Theorem). [II]]

Let $t_{i}(\bar{x}), i=1, \cdots, 6$. be terms, where $\bar{x}$ denotes the sequence $\left\langle x_{1}, \cdots x_{n}\right\rangle, x_{i}$ being varaibles. Let $\mathcal{V}$ be a subvariety of $\mathcal{I}$.

If

$$
\mathcal{V} \cap \mathcal{I}_{2,0} \models\left(t_{1}(\bar{x}) \rightarrow t_{2}(\bar{x})\right) \rightarrow t_{3}(\bar{x}) \approx\left(t_{4}(\bar{x}) \rightarrow t_{5}(\bar{x})\right) \rightarrow t_{6}(\bar{x}),
$$

then

$$
\mathcal{V} \models\left(t_{1}(\bar{x}) \rightarrow t_{2}(\bar{x})\right) \rightarrow t_{3}(\bar{x}) \approx\left(t_{4}(\bar{x}) \rightarrow t_{5}(\bar{x})\right) \rightarrow t_{6}(\bar{x})
$$


We are now ready to present the main result of this paper (i.e., Theorem $\mathbb{L} .4$ of Introduction).

Theorem 4.2. Let $\mathbf{A} \in \mathcal{I}$ then $\mathbf{A}_{m j}$ satisfies the Birkhoff's identity:

$$
\text { (BR) } x \wedge(x \vee y) \approx x \vee(x \wedge y) .
$$

Proof. Apply Theorem 5.2 and Theorem 4.1 .

Recall that $\mathcal{S}=\mathcal{I}_{2,0} \cap \mathcal{M C}$. The following corollary, which characterizes the implication zroupoids $\mathbf{A}$ for which $\mathbf{A}_{m j}$ is a Birkhoff system, is an improvement on [9, Theorem 7.3].

Corollary 4.3. Let $\mathbf{A} \in \mathcal{I}$. Then the algebra $\mathbf{A}_{m j}$ is a Birkhoff system if and only if $\mathbf{A} \in \mathcal{S}$.

Proof. Let $\mathbf{A}_{m j}$ be a Birkhoff system, then $\mathbf{A}$ satisfies (MC) and the identity: $x \wedge x \approx x$, which, in view of Lemma [2.], implies that $\mathbf{A}=x \approx x^{\prime \prime}$. Hence $\mathbf{A} \in \mathcal{S}$. For the converse, assume that $\mathbf{A} \in \mathcal{S}$. Then $\mathbf{A}$ satisfies the identities $x \wedge y \approx y \wedge x$ and $x \approx x^{\prime \prime}$. We know also by Theorem $\mathbb{L}$. that the operations $\wedge$ and $\vee$ are associative. Hence, from Theorem 4.2 (or Theorem [3.2) we conclude that $\mathbf{A}_{m j}$ is a Birkhoff system.

Recall that implication zroupoids that satisfy the associative identity:

$$
\text { (A) } x \rightarrow(y \rightarrow z) \approx(x \rightarrow y) \rightarrow z
$$

were called implication semigroups in [15.5]. Let $\mathcal{I S}$ denote the variety of implication semigroups. See [1.5] for the description of the lattice of subvarieties of $\mathcal{I S}$. The following special case of our main result may be of interest to the researchers in semigroup theory.

Corollary 4.4. Let $\mathbf{A} \in \mathcal{I S}$. Then the algebra $\mathbf{A}_{m j}$ satisfies the Birkhoff identity.

\subsection{Birkhoff bisemigroups and a restatement of the main theorem}

It follows from Theorem $4 \cdot \mathcal{Z}$ and Corollary 4.4 that, for every algebra $\mathbf{A} \in \mathcal{I} \backslash \mathcal{S}, \mathbf{A}_{m j}$ is a bisemigroup that is not a bisemilattice but satisfies the Birkhoff's identity. This suggests naturally a generalization of Birkhoff systems, which we will call "Birkhoff bisemigroups".

Definition 4.5. A bisemigroup $\mathbf{A}$ is a Birkhoff bisemigroup if $\mathbf{A} \models(B R)$.

To the best of our knowledge, the algebras defined in the above definition seem to be new. In this new terminology, we can now recast our main theorem as follows:

Theorem 4.6. If $\mathbf{A} \in \mathcal{I}$, then $\mathbf{A}_{m j}$ is a Birkhoff bisemigroup.

Thus, the class of algebras $\mathbf{A}_{\mathbf{m j}}$, where $\mathbf{A} \in \mathcal{I}$ provides a "large" class of examples of Birkhoff bisemigroups.

Another class of examples of Birkhoff bisemigroups arise from semigroups themselves as follows: Let $\mathbf{A}=\langle A, \wedge\rangle$ be a semigroup. Then the algebra $\langle A, \wedge, \wedge\rangle$ is clearly a bisemigroup and satisfies the Birkhoff identity trivially as the two binary operations are the same. Let us call such a bisemigroup arising from a semigroup "essentially a semigroup". We shall now improve and clarify Corollary 4.4. For this we need the following lemma:

Lemma 4.7. Let $\mathbf{A} \in \mathcal{I S}$. Then $\mathbf{A}$ holds:

$$
\begin{aligned}
& \text { 1. } 0 \rightarrow 0^{\prime} \approx 0, \\
& \text { 2. } 0 \rightarrow x^{\prime} \approx x^{\prime}, \\
& \text { 3. } 0^{\prime} \approx 0, \\
& \text { 4. } x \vee y \approx x \wedge y .
\end{aligned}
$$

Proof. Let $a, b \in A$. 
(प) By (A), we have

$$
0=0^{\prime \prime}=(0 \rightarrow 0) \rightarrow 0=0 \rightarrow(0 \rightarrow 0)=0 \rightarrow 0^{\prime} .
$$

(匹)

$$
\begin{aligned}
& a^{\prime}=a \rightarrow 0 \\
& =a \rightarrow\left(0 \rightarrow 0^{\prime}\right) \quad \text { by (四) } \\
& =(a \rightarrow 0) \rightarrow 0^{\prime} \quad \text { by }(\mathrm{A}) \\
& =\left[\left(0^{\prime \prime} \rightarrow a\right) \rightarrow\left(0 \rightarrow 0^{\prime}\right)^{\prime}\right]^{\prime} \quad \text { by }(\mathrm{I}) \\
& =\left[(0 \rightarrow a) \rightarrow\left(0 \rightarrow 0^{\prime}\right)^{\prime}\right]^{\prime} \\
& =\left[(0 \rightarrow a) \rightarrow 0^{\prime}\right] \rightarrow 0 \quad \text { by (四) } \\
& =(0 \rightarrow a) \rightarrow\left(0^{\prime} \rightarrow 0\right) \quad \text { by }(\mathrm{A}) \\
& =(0 \rightarrow a) \rightarrow 0^{\prime \prime} \\
& =(0 \rightarrow a) \rightarrow 0 \\
& =0 \rightarrow(a \rightarrow 0) \quad \text { by }(\mathrm{A}) \\
& =0 \rightarrow a^{\prime} \text {. }
\end{aligned}
$$

(B]) By (피) and (四), we have

$$
0^{\prime}=0 \rightarrow 0^{\prime}=0
$$

(困) By definition of $\vee$ and (A), we have

$$
\begin{aligned}
& a \vee b=a \rightarrow(0 \rightarrow(b \rightarrow(0 \rightarrow(0 \rightarrow(0 \rightarrow 0))))) \quad \text { by (B) } \\
& =a \rightarrow(0 \rightarrow(b \rightarrow 0)) \quad \text { by }(\mathbb{\nabla}) \\
& =a \rightarrow(b \rightarrow 0) \quad \text { by (B) } \\
& =a \rightarrow(b \rightarrow(0 \rightarrow 0)) \text { by }(\mathrm{A}) \\
& =a \rightarrow[(b \rightarrow 0) \rightarrow 0] \quad \text { by }(\mathrm{A}) \\
& =[a \rightarrow(b \rightarrow 0)] \rightarrow 0 \quad \text { def of } \wedge \\
& =a \wedge b \text {. }
\end{aligned}
$$

In view of the preceding lemma, Corollary 4.4 can be improved as follows.

Corollary 4.8. Let $\mathbf{A} \in \mathcal{I S}$. Then the algebra $\mathbf{A}_{m j}$ is essentially a semigroup.

\section{Conclusion}

In this paper it is proved that if $\mathbf{A} \in \mathcal{I}$, then the derived algebra $\mathbf{A}_{m j}:=\langle A ; \wedge, \vee\rangle$, where $a \wedge b:=\left(a \rightarrow b^{\prime}\right)^{\prime}$ and $a \vee b:=\left(a^{\prime} \wedge b^{\prime}\right)^{\prime}$, satisfies the Birkhoff's identity (BR): $x \wedge(x \vee y) \approx x \vee(x \wedge y)$. As consequences of this result, the implication zroupoids $\mathbf{A}$ whose derived algebras $\mathbf{A}_{m j}$ are Birkhoff systems are characterized, and a new variety of algebras called "Birkhoff bisemigroups" is defined, generalizing Birkhoff Systems.

Recall from [20] that the varieties of Boolean algebras, Kleene algebras, and De Morgan algebras can be viewed as subvarieties of $\mathcal{I}$. We conclude this paper with the following open problems:

PROBLEM 1: Find the regularization ([[18], for the definition) of the variety of Boolean algebras.

PROBLEM 2: Find the regularization of the variety of Kleene algebras. 
PROBLEM 3: Find the regularization of the variety of De Morgan algebras.

PROBLEM 4: Investigate Birkhoff bisemigroups; in particular, describe the structure of the lattice of subvarieties of the variety of Birkhoff bisemigroups.

The variety $\mathcal{I}$ seems to share some common properties with the variety of bounded BCK-algebras. For example, an unpublished result of ours says that $\mathcal{I}$ has the Glivenk property; that is, every algebra satisfies the identity: $(\mathrm{G}):(x \rightarrow y)^{\prime \prime} \approx x^{\prime \prime} \rightarrow y^{\prime \prime}$. For an analogous property of bounded BCK-algebras, see, for example, [6]. This leads us to raise the following problem.

PROBLEM 5: Explore the relationship between implication zroupoids and BCK-algebras.

Another unpublished result of ours is that the involutive algebras in the variety $\mathcal{I}$ satisfy the identity: $(x \oplus y) \rightarrow z \approx x \rightarrow(y \rightarrow z)$, if we take $\wedge$ to be the same as $\oplus$, which is also an axiom of hoops (see, for example, [4] ). This suggests that there is a connection between the variety of implication zroupoids and that of hoops, thus leading to the next problem.

PROBLEM 6: Investigate further connections between the variety of implication zroupoid and that of hoops.

\section{Acknowledgements}

The first author wants to thank the institutional support of CONICET (Consejo Nacional de Investigaciones Científicas y Técnicas) and Universidad Nacional del Sur. The authors also wish to acknowledge that [Mc] was a useful tool during the research phase of this paper.

The authors wish to dedicate this work to children and their families who fight against cancer.

\section{References}

[1] R. Balbes, P.H. Dwinger, Distributive lattices, University of Missouri Press, Columbia, 1974.

[2] B.A. Bernstein, A set of four postulates for Boolean algebras in terms of the implicative operation, Transactions of the American Mathematical Society, 36 (1934), 876-884.

[3] G. Birkhoff, Lattice theory, Second ed., American Mathematical Society Colloquium Publications, 25, American Mathematical Society, Providence, R.I., 1948.

[4] R.A. Borzooei, M. A. Kologani, Results on hoops, Journal of Hyper Algebraic Structures and Logical algebras, 1(1) (2020), 61-77.

[5] S. Burris, H.P. Sankappanavar, A course in universal algebra, Springer-Verlag, New York, 1981.

[6] D. Busneag, D. Piciu, M. Istrata, The Belluce lattice associated with a bounded BCK-algebra, Journal of Hyper Algebraic Structures and Logical algebras, 2(1) (2021), 1-16.

[7] J.M. Cornejo, H.P. Sankappanavar, Order in implication zroupoids, Studia Logica, 104(3) (2016), 417453.

[8] J.M. Cornejo, H.P. Sankappanavar, Semisimple varieties of implication zroupoids, Soft Computing, 20(3) (2016), 3139-3151.

[9] J.M. Cornejo, H.P. Sankappanavar, On implicator groupoids, Algebra Universalis, 77(2) (2017), 125146. 
[10] J.M. Cornejo, H.P. Sankappanavar, On derived algebras and subvarieties of implication zroupoids, Soft Computing, 21(23) (2017), 6963-6982.

[11] J.M. Cornejo, H.P. Sankappanavar, Symmetric implication zroupoids and identities of Bol-Moufang type, Soft Computing, 22 (2018), 4319-4333.

[12] J.M. Cornejo, H.P. Sankappanavar, Implication zroupoids and identities of associative type, Quasigroups and Related Systems, 26 (2018), 13-34.

[13] J.M. Cornejo, H.P. Sankappanavar, Symmetric implication zroupoids and weak associative identities, Soft Computing, 23(6) (2019), 6797-6812.

[14] J.M. Cornejo, H.P. Sankappanavar, Semi-distributivity and whitman property in implication Zroupoids, Mathematica Slovaka, (2021), 10 pages.

[15] S.V. Gusev, H.P. Sankappanavar, B.M. Vernikov, Implication semigroups, Order, 37 (2020), 1-7.

[16] J. Harding, A. Romanowska, Varieties of Birkhoff systems, Part I, Order, 34 (2017), 45-68.

[17] W. McCune, Prover 9 and Mace 4, (2005-2010). http://www.cs.unm.edu/mccune/prover9/

[18] J. Plonka, On distributive quasilattices, Fundamenta Mathematicae, 60 (1967), 191-200

[19] H. Rasiowa, An algebraic approach to non-classical logics, North-Holland, Amsterdam, 1974.

[20] H.P. Sankappanavar, De Morgan algebras: New perspectives and applications, Scientia Mathematicae Japonicae, 75(1) (2012), 21-50. 